\title{
Fear of Falling and Cognitive Impairments in Elderly People with Hip Fractures
}

\author{
Mari Kasai ${ }^{a}$ Kenichi Meguro ${ }^{a}$ Hiroshi Ozawa $^{b}$ Keiichi Kumai ${ }^{a}$ \\ Hideki Imaizumi $^{c}$ Hanae Minegishi $^{\text {b Hideki Oid }}{ }^{d}$ Akira Oizumi $^{\mathrm{e}}$ \\ Masahiro Yamashiro ${ }^{f}$ Michimasa Matsuda ${ }^{f}$ Masahiko Tanakag Eiji Itoi $^{\text {h }}$ \\ ${ }^{a}$ Geriatric Behavioral Neurology, Tohoku University CYRIC, Sendai, Japan; ${ }^{b}$ Department of \\ Orthopaedic Surgery, Faculty of Medicine, Tohoku Medical and Pharmaceutical University, \\ Sendai, Japan; 'Department of Orthopaedic Surgery, Osaki Citizen Hospital, Osaki, Japan; \\ ${ }^{\mathrm{d}}$ Department of Orthopaedic Surgery, Tome Citizen Hospital, Tome, Japan; ${ }^{\mathrm{e}}$ Department \\ of Orthopaedic Surgery, Kurihara Central Hospital, Kurihara, Japan; ' Department of \\ Orthopaedic Surgery, Matsuda Hospital, Sendai, Japan; ${ }^{9}$ Department of Orthopaedic

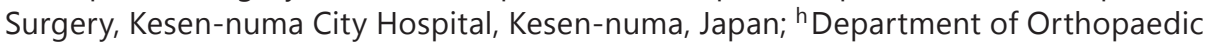 \\ Surgery, Tohoku University Graduate School of Medicine, Sendai, Japan
}

\section{Keywords}

Cognitive impairments · Fear of falling · Hip fractures · Prevalence - Dementia

\begin{abstract} ments.

Background/Aim: The purpose of this study was to investigate the estimated prevalence of dementia and the relationship between cognitive impairment and fear of falling in patients with hip fractures. Methods: Analysis 1 included 100 patients with hip fractures. Analysis 2 included a subgroup of subjects with $\geq 75$ years of functional independence: 46 patients with hip fractures and 46 control subjects without hip fractures, and presence or absence of dementia. We used an informant-rated questionnaire including the AD8 for screening for dementia, the Barthel Index for assessing activities of daily living, and the Short Falls Efficacy Scale-International (FES-I) for assessing fear of falling. Results: The estimated prevalence of dementia was $66 \%$ in patients with hip fractures. There were significant fracture and dementia effects, with significant covariate effects of age and gender on the Short FES-I scores. Conclusion: Our results suggested that more than two-thirds of patients with hip fractures had dementia. Fear of falling may reflect not only physical functions but also cognitive impair- 
Kasai et al.: Fear of Falling and Cognitive Impairments in Elderly People with Hip Fractures

\section{Introduction}

In patients with hip fractures, specialized rehabilitation for dementia might contribute to reduce postoperative complications and functional decline, which is a risk factor for institutionalization and higher mortality [1,2]. Early donepezil treatment and specialized physical therapy for very mild Alzheimer disease (AD) improved the patient's ability to learn cane gait and decreased the number of falls in a case report [3]. The early detection of dementia in patients with hip fractures has possibilities for a great beneficial effect on postoperative treatment and rehabilitation. According to a systematic review by Seitz et al. [4], the estimated prevalence of dementia in patients with hip fractures was 19.2\% (95\% confidence interval: $11.4-30.6 \%$ ) and the estimated prevalence of cognitive impairment was $41.8 \%$ (95\% confidence interval: 37.0-46.8\%). The systematic review described various Western countries' data such as the USA, the UK, Canada, etc. [4]. However, in previous reports, the estimated prevalence rate of dementia or cognitive impairment in patients with hip fractures varied widely. Also, there was no study of the estimated prevalence of dementia in Asian countries such as Japan.

The main cause of hip fractures was simple falls in approximately 78\% [5]. A previous systematic review reported various independent risk factors with falling such as physical and psychological factors. In the systematic review, the physical risk factors for falling included physical disability, visual and hearing impairments, walking aid use, etc. [6], whereas the psychological risk factors for falling included only 3 domains: cognitive impairment [6], depression [6], and fear of falling [6,7]. Focusing on symptoms of dementia, cognitive impairment was a core symptom of dementia [8] and depression was a common symptom of the behavioral and psychological symptoms of dementia $[9,10]$. Anxiety and fear regarding the future were types of psychological symptoms of dementia $[9,10]$. However, fear of falling has not been reported as a symptom of dementia, and the relationship between dementia and fear of falling was not clear. Recently, a few studies of fear of falling in patients with dementia have been published. A previous study reported that patients with AD and mild cognitive impairment (MCI) had more concerns about falling than cognitively healthy subjects [11]. However, the relationship between cognitive impairment and fear of falling in patients with hip fractures was not clear.

The purpose of this study was to investigate the estimated prevalence of dementia and the relationship between cognitive impairment and fear of falling in patients with hip fractures.

\section{Methods}

\section{Study Protocol}

Figure 1 shows the study protocol of this research. In Analysis 1, we calculated the estimated prevalence of dementia in 100 patients with hip fractures using the AD8 for screening of dementia $[12,13]$. We used the Barthel Index [14] for the functional evaluation of basic activities of daily living (ADL), and a score of $\geq 80$ was operationally defined as functionally independent in this study. To exclude the effects of age and physical dysfunction, we limited the subjects using the inclusion criteria: age $\geq 75$ years and Barthel Index scores $\geq 80$ in Analysis 2. As a scale of fear of falling, we used the Short Falls Efficacy Scale-International (FES-I) [15] for elderly people with/without hip fractures. We excluded 1 subject because of a lack of data on the Short FES-I. The subjects of Analysis 2 included 46 patients with hip fractures and 46 control subjects without hip fractures controlled for age, gender, Barthel Index scores, and presence or absence of dementia. 
Kasai et al.: Fear of Falling and Cognitive Impairments in Elderly People with Hip Fractures

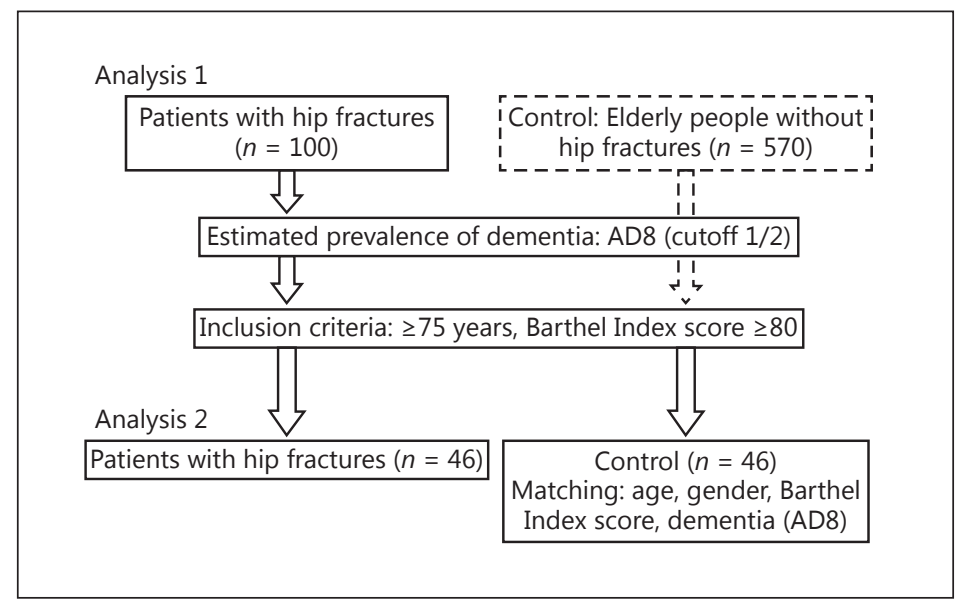

Fig. 1. Study protocol. In Analysis 1, we estimated the prevalence of dementia in 100 patients with hip fractures using the AD8 (cutoff score $\geq 2$, dementia). To exclude the effects of age and physical dysfunction, we limited the subjects: age $\geq 75$ years and Barthel Index scores $\geq 80$ (independent level in basic activities of daily living) in Analysis 2. The subjects of Analysis 2 included 46 patients with hip fractures and 46 control subjects without hip fractures matched for age, gender, Barthel Index scores, and presence or absence of dementia. We used the Short Falls Efficacy Scale-International (FES-I) for assessing fear of falling in elderly people with/without hip fractures.

\section{Analysis 1: Estimated Prevalence of Dementia in Patients with Hip Fractures}

Participants 1: Group with Hip Fractures

The group with hip fractures included 100 inpatients with hip fractures who were suitable for surgery and admitted to the orthopedics ward from December 2014 to June 2016 in Miyagi Prefecture in northern Japan. Of 111 enrolled patients during the period, 100 patients and their family members agreed to participate; we could not gain agreement with 11 patients' families for personal reasons. In this study, the Division of Geriatric Behavioral Neurology, CYRIC Tohoku University, integrated and analyzed all participating patients' data. We recruited the patients from 5 hospitals: Department of Orthopaedic Surgery, Osaki Citizen Hospital $(n=49)$, Department of Orthopaedic Surgery, Tohoku Medical and Pharmaceutical University Hospital $(n=33)$, Department of Orthopaedic Surgery, Tome Citizen Hospital $(n=8)$, Department of Orthopaedic Surgery, Kurihara Central Hospital $(n=5)$, Department of Orthopaedic Surgery, Matsuda Hospital $(n=3)$, and Department of Orthopaedic Surgery, Kesen-numa City Hospital $(n=2)$, Japan. In all patients with hip fractures, the mean age was 81.1 years (standard deviation [SD] 10.2), the percentage of women was $73 \%$, and the mean Barthel Index score was 81.4 (SD 26.6). In these patients, the ratio of neck fracture type of hip fractures in current injury was $41 \%$, and the ratio of trochanteric fracture type was $59 \%$. The mean length of time from injury to answering this questionnaire was 8.9 days (SD 9.1).

Procedure 1: Estimated Prevalence of Dementia

We calculated the estimated prevalence rate of dementia in patients with hip fractures using the AD8 for screening dementia. We used the informant-rated questionnaire of health state before the injury including the AD8 for patients with hip fractures and their family members or caregivers. This questionnaire included the AD8 for estimating the prevalence of dementia (AD8 scores $\geq 2$, with dementia) $[12,13]$. 
Kasai et al.: Fear of Falling and Cognitive Impairments in Elderly People with Hip Fractures

Table 1. Characteristics of patients with and without hip fractures

\begin{tabular}{|c|c|c|c|c|c|c|c|}
\hline & \multicolumn{2}{|c|}{ Group with hip fractures } & \multicolumn{2}{|c|}{$\begin{array}{l}\text { Control group } \\
\text { (without hip fractures) }\end{array}$} & \multirow{2}{*}{$\begin{array}{l}\text { Fractures } \\
\text { effect } \\
\text { ( } p \text { value) }\end{array}$} & \multirow{2}{*}{$\begin{array}{l}\text { Dementia } \\
\text { effect } \\
\text { ( } p \text { value) }\end{array}$} & \multirow{2}{*}{$\begin{array}{l}\text { Inter- } \\
\text { action } \\
(p \text { value })\end{array}$} \\
\hline & $\begin{array}{l}\text { with } \\
\text { dementia }\end{array}$ & $\begin{array}{l}\text { without } \\
\text { dementia }\end{array}$ & $\begin{array}{l}\text { with } \\
\text { dementia }\end{array}$ & $\begin{array}{l}\text { without } \\
\text { dementia }\end{array}$ & & & \\
\hline Patients, $n$ & 34 & 12 & 34 & 12 & & & \\
\hline Sex (male/female), $n$ & $10 / 24$ & $2 / 10$ & $10 / 24$ & $2 / 10$ & & 684 & \\
\hline Age, years & $84.3(5.5)$ & $83.6(6.2)$ & $83.9(5.1)$ & $83.4(5.9)$ & 0.842 & 0.652 & 0.943 \\
\hline Barthel Index scores & $95.7(6.4)$ & $99.6(1.4)$ & $94.9(7.1)$ & $99.6(1.4)$ & 0.754 & 0.003 & 0.754 \\
\hline
\end{tabular}

Age and Barthel Index scores show means (standard deviation). Group with dementia, AD8 score $\geq 2$; group without dementia, AD8 score <2. Barthel Index: basic activities of daily living assessment, score range, 0 (dependent) to 100 (perfect independent). The control group (without hip fractures) matched age, gender, Barthel Index, and presence or absence of dementia. Statistical analyses: age and Barthel Index, two-way ANOVA; gender, $\chi^{2}$ test.

\section{Analysis 2: Fear of Falling in Elderly People with/without Hip Fractures}

Participants 2: Elderly People with/without Hip Fractures

Group with Hip Fractures. We limited 100 patients with hip fractures according to the inclusion criteria age $\geq 75$ years and Barthel Index scores $\geq 80$ to exclude the effects of age and physical dysfunction. We excluded 20 patients aged $\leq 74$ years and 23 patients with Barthel Index scores $<80$. Additionally, we excluded 1 subject who lacked data for the Short FES-I. Finally, the subjects of Analysis 2 included 46 patients with hip fractures.

Group without Hip Fractures (Control Group). A total of 590 of 1,252 subjects aged $\geq 75$ years agreed to take part in the Kurihara Project [16] living in Kurihara in Miyagi Prefecture in northern Japan, from October 2008 to December 2010. Of these 590 subjects, 20 were excluded: 3 subjects had a past history of hip fractures and 17 subjects did not complete the questionnaire of this study. Of 570 community dwellers, we selected 46 control subjects without hip fractures, matching the patients in the group with hip fractures in age, gender, Barthel Index scores, and presence or absence of dementia.

Table 1 shows the characteristics of patients with and without hip fractures. The group with hip fractures included 46 patients, and the group without hip fractures included 46 subjects. In age, there was no significant fracture effect $(F=0.0, p=0.842)$ and no dementia effect $(F=0.2, p=0.652)$ without interaction $(F=0.0, p=0.943)$ for two-way analysis of variance (ANOVA). In gender, there was no significant difference among the 4 groups $\left(\chi^{2}=\right.$ $1.5, p=0.684$ ) for $\chi^{2}$ tests. In Barthel Index scores, there was no significant fracture effect $(F=0.1, p=0.754)$, but a significant dementia effect $(F=9.3, p=0.003)$ without interaction $(F=0.1, p=0.754)$ for two-way ANOVA.

Procedure 2: Fear of Falling in Elderly People with/without Hip Fractures

We investigated fear of falling in elderly people with/without hip fractures using the Short FES-I. We used two-way analysis of covariance (ANCOVA) for the Short FES-I scores [2 hip fracture effects (with hip fractures vs. without hip fractures) $\times 2$ dementia effects (with dementia vs. without dementia)]. As an additional analysis, we compared the self-rated version with the informant-rated version of the Short FES-I scores in patients with hip fractures using one-way repeated-measures ANOVA. We used the IBM SPSS statistics version 22 software for the statistical analysis (IBM Corp., Armonk, NY, USA). 
Kasai et al.: Fear of Falling and Cognitive Impairments in Elderly People with Hip Fractures

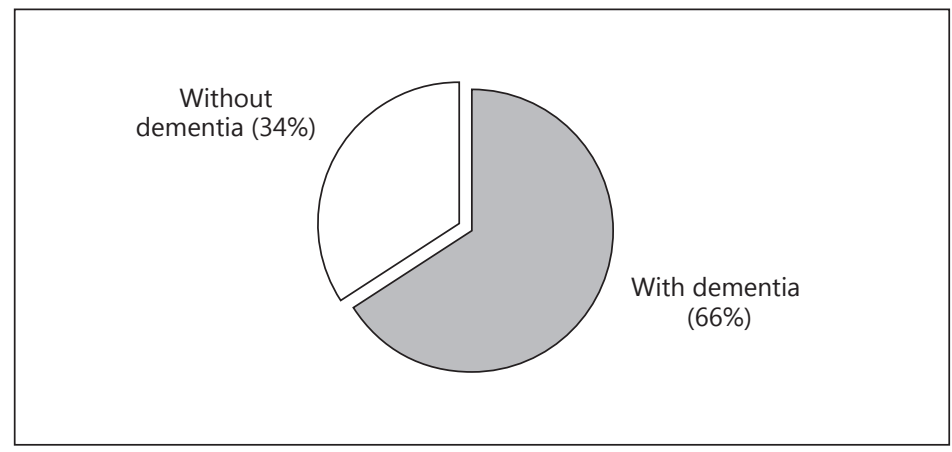

Fig. 2. Estimated prevalence of dementia in patients with hip fractures. The gray area shows the estimated prevalence rate of the group with dementia, and the white area shows the prevalence rate of the group without dementia in 100 patients with hip fractures, using the AD8 for screening dementia. Group with dementia, AD8 score $\geq 2$; group without dementia, AD8 score $<2$.

\section{Questionnaire}

In the group with hip fractures, we used the self-rated and informant-rated questionnaire regarding the state before the injury of patients with hip fractures from both patients and their family or caregivers. In the control group, we used the same informant-rated questionnaire on the health state of subjects from their family or caregivers. This questionnaire included the AD8 for estimating prevalence of dementia [12, 13], the Barthel Index [14] for assessing physical disabilities of ADL, and the Short FES-I [15] (informant-rated) for assessing fear of falling.

$A D 8$. The AD8 is a brief, sensitive informant interview to detect dementia $[12,13,17]$. The cutoff score of the Japanese version of the AD8 for screening for dementia was 1/2 (AD8 scores $\geq 2$, with dementia), with a sensitivity of $88.4 \%$ and a specificity of $68.4 \%$ in a community-based study [13].

Barthel Index. We used the Barthel Index [14] for assessing functional disability of basic ADL for subjects with/without hip fractures. The Barthel Index consists of 10 items: feeding, bathing, grooming, dressing, bowels, bladder, toilet use, transfers, mobility, and stairs.

Short FES-I. The Short FES-I is a brief and common scale for assessing fear of falling in older people $[15,18]$. The Short FES-I [15] consists of 7 items with 4 answer options each. The 7 items of the Short FES-I included: getting dressed or undressed, taking a bath or shower, getting in or out of a chair, going up or down stairs, reaching for something above your head or on the ground, walking up or down a slope, and going out to a social event (e.g., religious service, family gathering, or club meeting) [15]. The 4 answer options were as follows: not at all concerned, somewhat concerned, fairly concerned, and very concerned [15]. The scoring range was from 7 (no concern about falling) to 28 (severe concern about falling) [15]. We used the 2010 Japanese translation of the FES-I by Kamide et al. [19]. The FES-I was a reliable assessment of fear of falling in patients after a hip fracture [20]. In the current study, we used both the self-rated and the informant-rated versions of the Short FES-I in patients with hip fractures, and we used the informant-rated version of the Short FES-I in subjects without hip fractures.

\section{Statement of Ethics}

Written informed consent was obtained from every participant and from their family members. The study was approved by the Ethics Committees of each hospital and Tohoku University Graduate School of Medicine. 
Kasai et al.: Fear of Falling and Cognitive Impairments in Elderly People with Hip Fractures

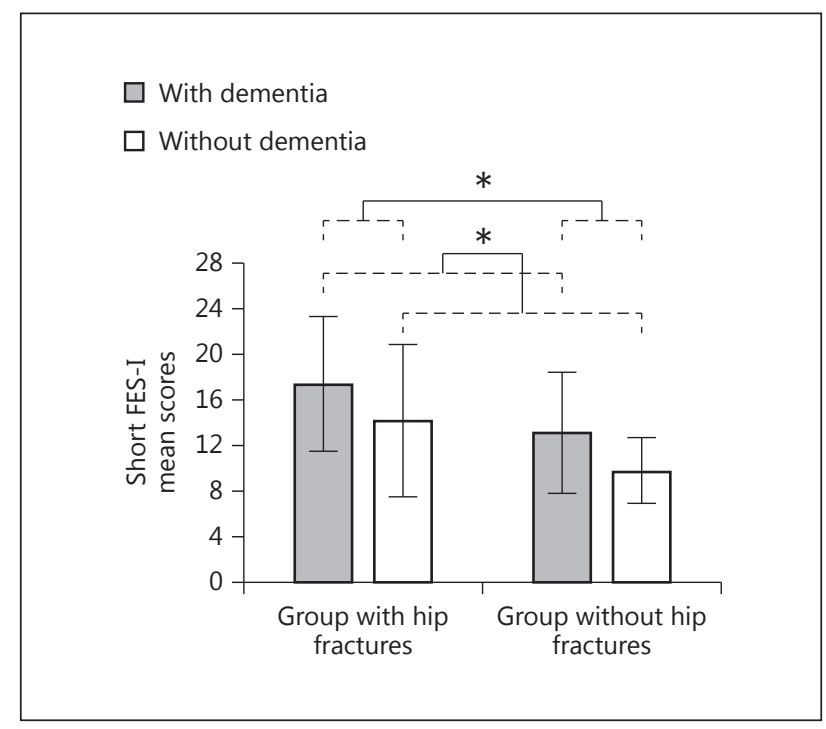

Fig. 3. Short Falls Efficacy Scale-International (FES-I) scores for assessing fear of falling in Analysis 2. The subjects of Analysis 2 included 46 patients with hip fractures and 46 control subjects without hip fractures matched for age, gender, Barthel Index scores, and presence or absence of dementia. The gray bars show patients with dementia, and the white bars show patients without dementia. The vertical line shows the mean scores of the Short FES-I. For the two-way analysis of covariance (ANCOVA), there was a significant hip fracture effect $(p<0.05)$ and a significant dementia effect $(p<0.05)$, but not a significant interaction $(p>0.05)$ between the two groups. There were significant covariate effects on age and gender $(p<0.05)$, but not on Barthel Index scores between the two groups $(p>0.05) . * p<0.05$.

\section{Results}

\section{Result 1: Estimated Prevalence of Dementia in Patients with Hip Fractures}

Figure 2 shows the estimated prevalence of dementia in patients with hip fractures. In 100 patients with hip fractures, the estimated prevalence rate of dementia was $66 \%(66 / 100)$, using the AD8 scale. Additionally, in a subgroup of subjects aged $\geq 75$ years, the estimated prevalence rate of dementia was $76 \%(61 / 80)$. In a subgroup of subjects aged $\geq 75$ years with a functionally independent level (Barthel Index score $\geq 80$ ), the estimated prevalence rate of dementia was $72 \%$ (34/47).

\section{Result 2: Fear of Falling in Elderly People with/without Hip Fractures}

Figure 3 shows the result of the Short FES-I scores for assessing fear of falling in Analysis 2 . In a subgroup of subjects aged $\geq 75$ years with a functionally independent level (Barthel Index score $\geq 80$ ), the group with hip fractures included 46 patients, and the group without hip fractures included 46 subjects without hip fractures, matched for age, gender, Barthel Index scores, and presence or absence of dementia. On the Short FES-I scores, there was a significant fracture effect $(F=13.9, p<0.001)$ and dementia effect $(F=7.1, p=0.009)$, but not an interaction $(F=0.0, p=0.953)$ between the two groups using the $2 \times 2$ two-way ANCOVA. There were significant covariate effects in age $(F=10.8, p=0.002)$ and gender $(F=11.7, p=$ $0.001)$, but not Barthel Index scores $(F=0.9, p=0.337)$ between the two groups. For post hoc tests, the group with fractures/with dementia had significantly more concerns about fear of falling than the group without fractures/with dementia ( $t$ test, $t=3.1, p=0.003$ ) and the group without fractures/without dementia ( $t$ test, $t=5.8, p<0.001)$. There was no significant difference between the other groups ( $p>0.05)$. 
For additional analysis, we compared the self-rated version with the informant-rated version of the Short FES-I scores in patients with hip fractures. We analyzed both the selfrated and the informant-rated versions of the Short FES-I in 44 patients with hip fractures, excluding 2 patients because of a lack of data for the self-rated version. The mean score of the self-rated version was 14.6 (SD 5.3) and that of the informant-rated version was 16.3 (SD 6.1) on the Short FES-I. There was no significant difference between the self-rated version and the informant-rated version in the Short FES-I scores $(F=3.5, p=0.067)$ using one-way repeatedmeasures ANOVA.

\section{Discussion}

We used the informant-rated questionnaire of health state before the injury from the family or caregiver of the patients in the group with hip fractures. We estimated that the prevalence rate of dementia in patients with hip fractures was $66 \%$. We limited the subjects using the inclusion criteria in Analysis 2: age $\geq 75$ years and Barthel Index scores $\geq 80$. The subjects included 46 patients with hip fractures and 46 control subjects without hip fractures, matched for age, gender, Barthel Index scores, and presence or absence of dementia. We used the Short FES-I for assessing fear of falling for subjects with/without hip fractures. There were significant effects of fracture and dementia on the Short FES-I scores between the two groups. Fear of falling may reflect not only fractures but also cognitive functions.

Our estimated prevalence was higher than that in the previous report by Seitz et al. [4]. In the systematic review of patients with hip fractures, the estimated prevalence rate of dementia varied widely by subjects' age [4]. The prevalence of cognitive impairment was $9 \%$ in a report by Galanakis et al. [21], which was the "youngest" report in this review, with a mean age of 75 years. Meanwhile, in the same systematic review, the prevalence of cognitive impairment was $60 \%$ in the report by Goldstein et al. [22], which was the "oldest" report in this review, with a mean age of 84 years. When we limited subjects to $\geq 75$ years of age (mean age, 85 years), the estimated prevalence rate of dementia was $76 \%$, which was higher than that in the previous reports. It was suggested that the subjects' age was a factor affecting the estimated prevalence rate of dementia in patients with hip fractures.

In this study, we used the AD8, which was a brief and informant-rated scale for screening of dementia. The Japanese version of the AD8 [13] had good sensitivity, but not good specificity, using standard cutoff scores. Since our subjects with dementia using the AD8 might include not only patients with dementia but also patients with $\mathrm{MCI}$, our estimated prevalence rate of dementia was higher than that in the previous reports.

We did not diagnose the causes of dementia in our patients, since the patients did not undergo head magnetic resonance imaging (MRI) scans. As discussion data, however, we used the Hachinski Ischemic Score [23] for classification of dementia in patients with hip fractures. Of 66 patients with dementia after hip fractures, 28 patients had "AD" type (Hachinski Ischemic score $\leq 4$ ), 22 patients had "mixed" type (Hachinski Ischemic score 5 or 6 ), and 16 patients had "vascular dementia" type (Hachinski Ischemic score $\geq 7$ ) in this study. The number of patients with "AD" type was higher than that of patients with "vascular dementia" type in our population.

The patients with both fractures and dementia had significantly more concerns about fear of falling than patients without fractures on the Short FES-I scores in Analysis 2. This result suggests that fear of falling in patients with hip fractures might reflect not only physical dysfunctions but also cognitive impairments. In the additional analysis, there was no significant difference between the self-rated and the informant-rated versions of the Short FES-I. However, a previous study showed a significant difference between the self-rated and the 
Kasai et al.: Fear of Falling and Cognitive Impairments in Elderly People with Hip Fractures

informant-rated versions of the Short FES-I [24]. One reason for the opposite outcome may be the difference in the rate of dementia (10\% in the previous study [24] vs. $66 \%$ in our study). This suggests that the performance on the Short FES-I might be affected by cognitive impairments.

Our study described that patients with dementia had more concerns about fear of falling before the injury than patients without dementia. Our results suggest that "fear of falling" in patients with dementia might be a type of phobia, which is a psychological symptom of dementia. We propose "fear of falling" to be added as a type of "anxiety and phobias" in the assessment of psychological symptoms of dementia. Fear of falling may be a kind of cue to start giving care to patients with dementia. Generally, most patients with dementia have impairment in judgment, and they might receive caregiver's care including "monitoring" for patients. This result suggests that, as a proposal, we could add the stage of "monitoring" before physical assistance to the item of self-care in clinical dementia scales, such as the Clinical Dementia Rating [25]. We need to investigate the relationships between fear of falling and judgment, attention, and executive function in patients with hip fractures.

This study has several limitations. We used the AD8 for assessing dementia in this study. The AD8 for community-dwelling elderly had high sensitivity and slightly low specificity for detecting dementia in a previous study [13]. Our subjects might include not only patients with dementia but also several patients with MCI. We could not perform MRI scans in all subjects for the diagnosis of dementia and dementing diseases, and we could not analyze the severity of dementia with hip fractures in this study.

In conclusion, our results suggested that more than two-thirds of patients with hip fractures had dementia. Fear of falling may reflect not only physical functions but also cognitive impairments. Fear of falling in patients with dementia might be a type of phobia in a psychological symptom of dementia. In the future, we need to diagnose dementia and dementing diseases, and to improve specialized dementia of postoperative rehabilitation in patients with hip fractures. The present study contributes to the early detection of dementia and the improvement of the quality of long-term care in the future.

\section{Acknowledgements}

We are grateful to all medical staff in this study for their research cooperation: Department of Orthopaedic Surgery, Tohoku University School of Medicine (Dr. Toshimi Aizawa), The Osaki-Tajiri SKIP Center (Dr. Satoshi Yamaguchi), Department of Orthopedic Surgery, Osaki Citizen Hospital (Ms. Kazue Ujiie), Department of Orthopaedic Surgery, Tohoku Medical and Pharmaceutical University Hospital (Dr. Tokuhisa Sano), Department of Orthopaedic Surgery, Tome Citizen Hospital (Ms. Kazue Shinobu), Department of Orthopaedic Surgery, Kurihara Central Hospital, and Department of Orthopaedic Surgery, Kesen-numa City Hospital (Dr. Kentaro Ito).

We are grateful to all staff at the Division of Geriatric Behavioral Neurology, CYRIC, Tohoku University. We thank Dr. Kei Nakamura, Ms. Keiko Chida, Ms. Konomi Takahashi, Mr. Jiro Oonuma, and Dr. Hirohisa Koide for their assistance.

\section{Disclosure Statement}

None of the authors have conflicts of interest. 
Kasai et al.: Fear of Falling and Cognitive Impairments in Elderly People with Hip Fractures

\section{Funding Sources}

Lilly Research Grant Program for Bone and Mineral Research 2016, Japan Osteoporosis Foundation (Tokyo, Japan), provided funding for this study.

\section{References}

1 Seitz DP, Gill SS, Gruneir A, Austin PC, Anderson GM, Bell CM, Rochon PA: Effects of dementia on postoperative outcomes of older adults with hip fractures: a population-based study. J Am Med Dir Assoc 2014;15:334-341.

-2 Seitz DP, Gill SS, Austin PC, Bell CM, Anderson GM, Gruneir A, Rochon PA: Rehabilitation of older adults with dementia after hip fracture. J Am Geriatr Soc 2016;64:47-54.

-3 Kumai K, Nakamura K, Meguro K: Improved learning of sequential behavior during cane gait training or stair climbing after femoral neck fracture: an implication for donepezil for very mild Alzheimer's disease. Psychogeriatrics 2017; 17:144-145.

4 Seitz DP, Adunuri N, Gill SS, Rochon PA: Prevalence of dementia and cognitive impairment among older adults with hip fractures. J Am Med Dir Assoc 2011;12:556-564.

-5 Hagino H, Sakamoto K, Harada A, Nakamura T, Mutoh Y, Mori S, Endo N, Nakano T, Itoi E, Kita K, Yamamoto N, Aoyagi K, Yamazaki K; Committee on Osteoporosis of The Japanese Orthopedic Association: Nationwide onedecade survey of hip fractures in Japan. J Orthop Sci 2010;15:737-745.

-6 Deandrea S, Lucenteforte E, Bravi F, Foschi R, La Vecchia C, Negri E: Risk factors for falls in communitydwelling older people: a systematic review and meta-analysis. Epidemiology 2010;21:658-668.

7 Denkinger MD, Lukas A, Nikolaus T, Hauer K: Factors associated with fear of falling and associated activity restriction in community-dwelling older adults: a systematic review. Am J Geriatr Psychiatry 2015;23:72-86.

8 American Psychiatric Association: Diagnostic and Statistical Manual of Mental Disorders, Fourth Edition (DSM-IV). American Psychiatric Association, Washington, 1994.

-9 Reisberg B, Borenstein J, Salob SP, Ferris SH, Franssen E, Georgotas A: Behavioral symptoms in Alzheimer's disease: phenomenology and treatment. J Clin Psychiatry 1987;48(suppl):9-15.

10 Monteiro IM, Boksay I, Auer SR, Torossian C, Ferris SH, Reisberg B: Addition of a frequency-weighted score to the Behavioral Pathology in Alzheimer's Disease Rating Scale: the BEHAVE-AD-FW: methodology and reliability. Eur Psychiatry 2001;16(suppl 1):5s-24s.

-11 Borges Sde M, Radanovic M, Forlenza OV: Fear of falling and falls in older adults with mild cognitive impairment and Alzheimer's disease. Neuropsychol Dev Cogn B Aging Neuropsychol Cogn 2015;22:312-321.

12 Galvin JE, Roe CM, Powlishta KK, Coats MA, Muich SJ, Grant E, Miller JP, Storandt M, Morris JC: The AD8: a brief informant interview to detect dementia. Neurology 2005;65:559-564.

13 Meguro K, Kasai M, Nakamura K; Kurihara Project members: Reliability and validity of the Japanese version of the AD8 (in Japanese). Nihon Ronen Igakkai Zasshi 2015;52:61-70.

14 Mahoney FI, Barthel DW: Functional evaluation: the Barthel Index. Md State Med J 1965;14:61-65.

15 Kempen GI, Yardley L, van Haastregt JC, Zijlstra GA, Beyer N, Hauer K, Todd C: The Short FES-I: a shortened version of the Falls Efficacy Scale-International to assess fear of falling. Age Ageing 2008;37:45-50.

16 Meguro K, Tanaka N, Kasai M, Nakamura K, Ishikawa H, Nakatsuka M, Satoh M, Ouchi Y: Prevalence of dementia and dementing diseases in the old-old population in Japan: the Kurihara Project. Implications for long-term care insurance data. Psychogeriatrics 2012;12:226-234.

17 Galvin JE, Roe CM, Coats MA, Morris JC: Patient's rating of cognitive ability: using the AD8, a brief informant interview, as a self-rating tool to detect dementia. Arch Neurol 2007;64:725-730.

18 Yardley L, Beyer N, Hauer K, Kempen G, Piot-Ziegler C, Todd C: Development and initial validation of the Falls Efficacy Scale-International (FES-I). Age Ageing 2005;34:614-619.

19 Kamide N, Shiba Y, Takahashi K, Inaba Y, Haga H: Reliability and validity of the Falls Efficacy Scale International in Japanese community-dwelling elderly women. Sogo Rehabil 2010;38:1063-1069.

20 Visschedijk JH, Terwee CB, Caljouw MA, Spruit-van Eijk M, van Balen R, Achterberg WP: Reliability and validity of the Falls Efficacy Scale-International after hip fracture in patients aged $\geq 65$ years. Disabil Rehabil 2015;37: 2225-2232.

21 Galanakis P, Bickel H, Gradinger R, Von Gumppenberg S, Förstl H: Acute confusional state in the elderly following hip surgery: incidence, risk factors and complications Int J Geriatr Psychiatry 2001;16:349-355.

22 Goldstein FC, Strasser DC, Woodard JL, Roberts VJ: Functional outcome of cognitively impaired hip fracture patients on a geriatric rehabilitation unit. J Am Geriatr Soc 1997;45:35-42.

23 Hachinski VC, Iliff LD, Zilhka E, Du Boulay GH, McAllister VL, Marshall J, Russell RW, Symon L: Cerebral blood flow in dementia. Arch Neurol 1975;32:632-637.

24 Shen J, Hu F, Liu F, Tong P: Functional restriction for the fear of falling in family caregivers. Medicine (Baltimore) 2015;94:e1090.

25 Morris JC: The Clinical Dementia Rating (CDR): current version and scoring rules. Neurology 1993;43:24122414. 